\title{
Baseline spatial distribution of malaria prior to an elimination programme in Vanuatu
}

\author{
Heidi Reid*1, Andrew Vallely', George Taleo², Andrew J Tatem4, Gerard Kelly', lan Riley1, Ivor Harris³, lata Henri², \\ Sam lamaher ${ }^{2}$ and Archie CA Clements 1,5
}

\begin{abstract}
Background: The Ministry of Health in the Republic of Vanuatu has implemented a malaria elimination programme in Tafea Province, the most southern and eastern limit of malaria transmission in the South West Pacific. Tafea Province is comprised of five islands with malaria elimination achieved on one of these islands (Aneityum) in 1998. The current study aimed to establish the baseline distribution of malaria on the most malarious of the province's islands, Tanna Island, to guide the implementation of elimination activities.

Methods: A parasitological survey was conducted in Tafea Province in 2008. On Tanna Island there were 4,716 participants from 220 villages, geo-referenced using a global position system. Spatial autocorrelation in observed prevalence values was assessed using a semivariogram. Backwards step-wise regression analysis was conducted to determine the inclusion of environmental and climatic variables into a prediction model. The Bayesian geostatistical logistic regression model was used to predict malaria risk, and associated uncertainty across the island.

Results: Overall, prevalence on Tanna was 1.0\% for Plasmodium falciparum (accounting for 32\% of infections) and 2.2\% for Plasmodium vivax (accounting for $68 \%$ of infections). Regression analysis showed significant association with elevation and distance to coastline for P. vivax and P. falciparum, but no significant association with NDVI or TIR. Colinearity was observed between elevation and distance to coastline with the later variable included in the final Bayesian geostatistical model for P. vivax and the former included in the final model for $P$. falciparum. Model validation statistics revealed that the final Bayesian geostatistical model had good predictive ability.

Conclusion: Malaria in Tanna Island, Vanuatu, has a focal and predominantly coastal distribution. As Vanuatu refines its elimination strategy, malaria risk maps represent an invaluable resource in the strategic planning of all levels of malaria interventions for the island.
\end{abstract}

\section{Background}

In recent years, the momentum behind malaria elimination has gathered speed with thirty-nine countries across the world now making progress toward malaria elimination. One of the key strategies is to shrink the global malaria map from the endemic margins inward [1]. While some nations are committed to nationwide elimination, others are pursuing spatially progressive elimination within their borders. With support from international donors, the Ministry of Health in the Republic of Vanuatu has started to implement a malaria elimination pro-

* Correspondence: heidilouisereid@gmail.com

1 Pacific Malaria Initiative Support Centre (PacMISC), Australian Centre for International and Tropical Health (ACITH), School of Population Health, University of Queensland, Queensland, Australia

Full list of author information is available at the end of the article gramme in Tafea Province which is comprised of five islands, Fatuna, Aneityum, Erromango, Aniwa and Tanna (Figure 1). Interrupted malaria transmission has already been achieved on the island of Aneityum through the use of approaches such as mass drug administration and insecticide treated bed nets, and with the enthusiastic support of the local population [2]. Tafea Province represents the most southern and eastern limit of malaria in the South West Pacific and thus a strategic starting point for elimination activities.

While the optimal strategy for elimination is being debated, possible distinctions between control and elimination efforts are the geographical scale and intensity of key interventions [3]. During the control phase, interventions tend to be widely applied to the target areas, often 


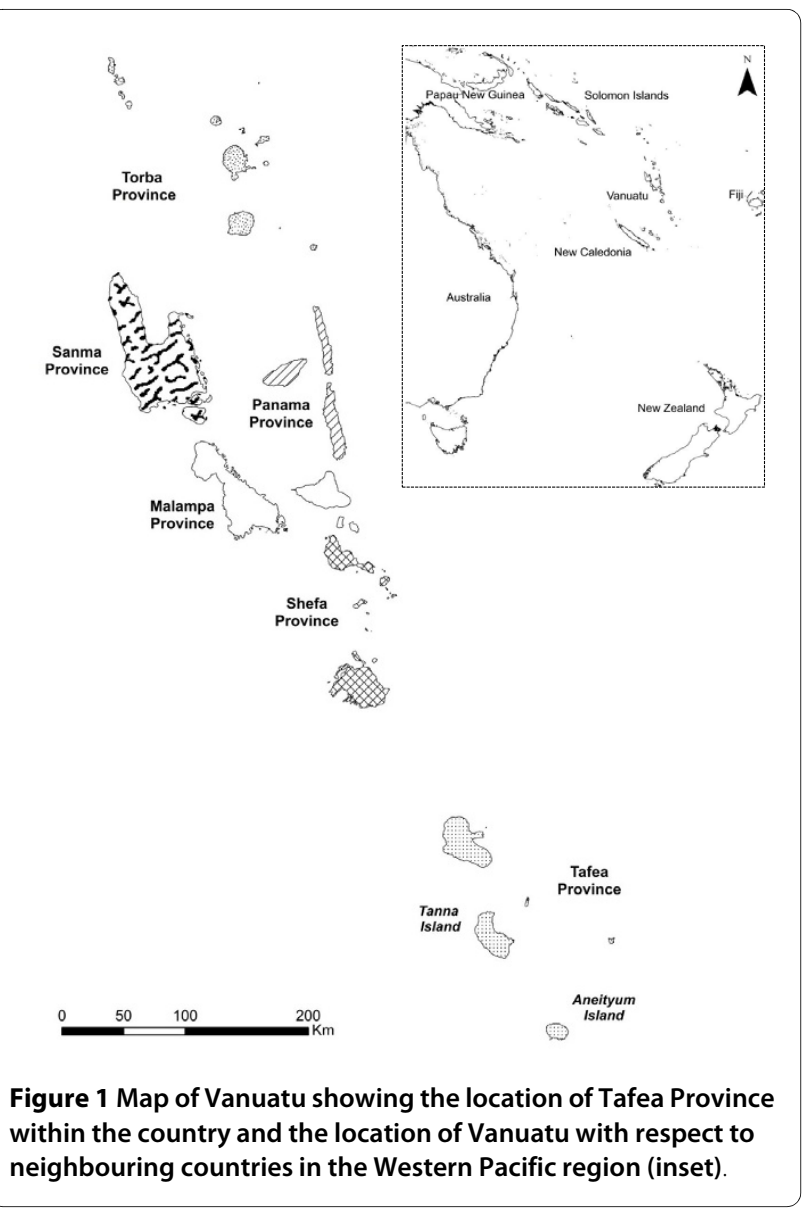

with similar strategies between zones of varying endemicity. As the prevalence declines remaining transmission is increasingly restricted to specific geographical foci and more precision in the application of interventions is needed, with more intense targeting of resources to identify and eliminate the last remaining sites of transmission and/or resistance [4]. An effective elimination campaign must be capable of identifying these foci. Mapping offers great potential and the greatest need for malaria maps is at the periphery of stable, endemic areas where there is often less empirical information regarding risks and intensity of infection [5].

The advancement of geographical information systems (GIS) and spatial statistics has greatly improved our understanding of malaria dynamics, including its dependence on ecological factors [5-12]. More recently, Bayesian geostatistics has been embraced for disease mapping with the advantage that both environmental covariates and spatial autocorrelation are able to be estimated simultaneously and full posterior distributions produced, which can be used to quantify uncertainties in parameters of interest (e.g. predicted prevalence of infection)[13]. Spatial prediction models have been used to produce malaria risk maps at national $[11,14-19]$ subcontinental [20-22] and global scales [23,24].

The aim of this present study was to produce accurate, validated predicted prevalence maps for $P$. vivax and $P$. falciparum on Tanna Island, Vanuatu. Additionally, it is envisaged that the maps be used to spatially define an implementation strategy for malaria elimination in Tanna Island. The applicability of the methods and the implications of the results are discussed in the context of malaria elimination strategies, which are beginning to take shape.

\section{Methods}

\section{Survey data}

Data pertaining specifically to Tanna Island $(\mathrm{N}=4763)$ were extracted from the results of a parasitological survey conducted in Tafea Province in 2008 by the National Vector Borne Disease Control Program (VBDCP), Vanuatu, in collaboration with the Pacific Malaria Initiative Support Centre (PacMISC), a Brisbane-based consortium consisting of the School of Population Health at the University of Queensland; the Australian Army Malaria Institute (AMI); and the Queensland Institute of Medical Research (QIMR)[25]. Within Tanna, the school-based survey covered all 80 schools on the island. Blood samples were collected from children between two and 12 years of age by finger prick using a lancet and the samples were examined microscopically for malaria parasites. Dried blood spot specimens were also collected and transported to the AMI in Brisbane for analysis by polymerase chain reaction (PCR), considered the gold standard for malaria diagnosis, the results of which were used to develop the spatial models presented in this report.

Each child was interviewed to collect information such as school [ $\mathrm{N}=80]$, home village [ $\mathrm{N}=233]$ and village in which they usually sleep ('sleep' village, $\mathrm{N}=233$ ). Village coordinates were not taken at the time of the survey but school, home and sleep villages were later matched to a government list provided by the Vanuatu Ministry of Lands from a 1999 census. To most accurately match infection to place of transmission, sleep village was used as the geographical reference because this was considered a more accurate representation of exposure sites than home or school village. For sleep villages not able to be geo-referenced, the home and then school village were used as the geographical reference. If villages were not included in the census list ( $4 \%$ of children), the local malaria control officer on Tanna Island was consulted to determine the closest listed village to the sleep village and this was chosen as the geographical reference. A total of 4,716 children (99\%) could be geo-located in this manner to a total of 220 'sleep', 'home' or 'school' villages.

Four villages in the Green Hill area in the north of the island (circled in Figure 2) showed an unexpectedly high 


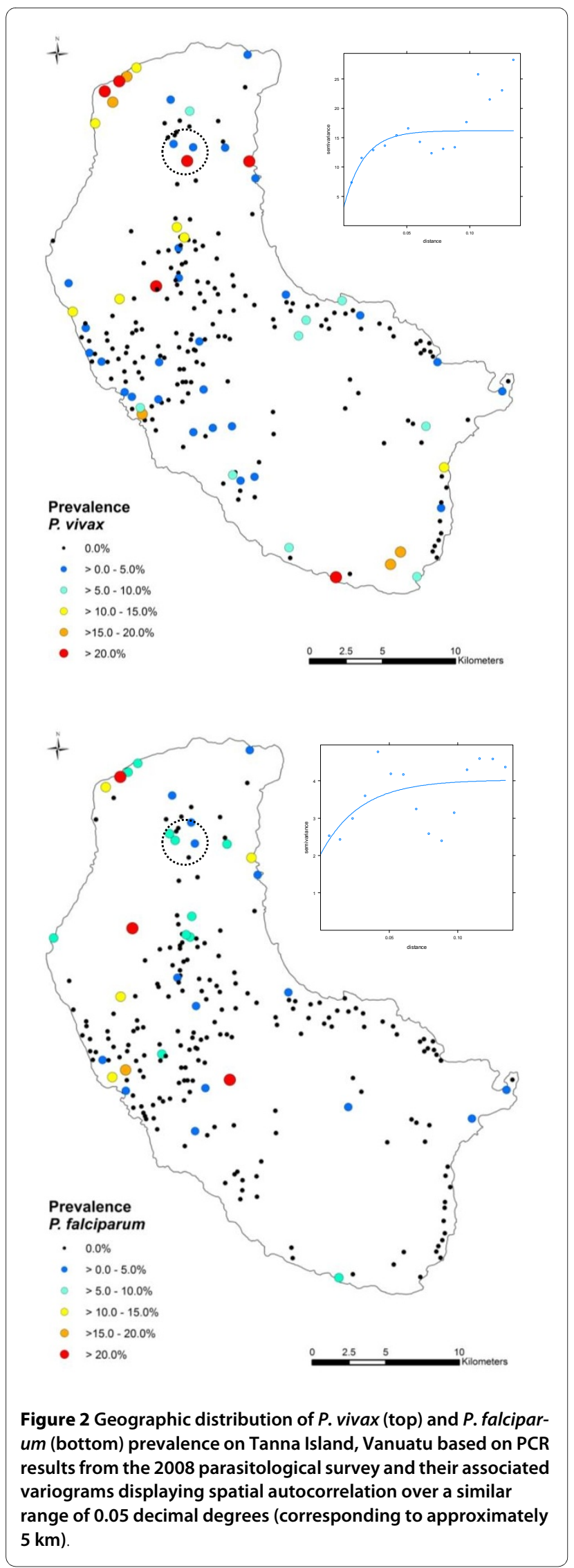

proportion of malaria-positive individuals given their inland location. As this finding was hypothesized to be an artefact of the post-survey geo-referencing method, these four villages were removed during a secondary analysis to assess their impact on the significance of environmental variables.

\section{Analysis of spatial structure}

Statistical analyses of spatial structure in the prevalence data were done in $\mathrm{R}$ version 2.9.0 (The $\mathrm{R}$ foundation for statistical computing) using the GeoR package. For these analyses the sample variogram was defined as:

$$
\gamma(h)=\frac{1}{2 n(h)} \sum_{i=1}^{n(h)}\left(p\left(x_{i}\right)-p\left(x_{i}+h\right)\right)^{\wedge} 2
$$

Where $p\left(x_{i}\right)$ represents a value of prevalence observed at location $x_{i}$ and $p\left(x_{i}-h\right)$ represents a second observation at a distance $h$ from the first. By discretising lags into a series of bins of width $b$, such that each value of $h$ actually represents a distance interval $h \pm 1 / 2 b$, semi-variances are computed as the mean semi-variance amongst the set of $n(h)$ pairs of observations separated be distances within that interval [24].

\section{Assembling and testing ecological and climate variables} Images from Landsat Enhanced Thematic Mapper (ETM) were downloaded from the United States Geological Survey (USGS) Earth Resources Observation and Science Center [26]. These images were processed to develop estimates of normalized difference vegetation index (NDVI) [27] representing the amount of vegetation per 30 metre spatial resolution pixel and Thermal Infra-Red (TIR) as a surrogate for surface temperature at $60 \mathrm{~m} \mathrm{spa-}$ tial resolution.

Gridded elevation data at $90 \mathrm{~m}$ spatial resolution were obtained from the Shuttle Radar Topography Mission digital elevation dataset, processed and made available at the Consultative Group on International Agricultural Research Consortium for Spatial information [28]. Finally distance to coast was calculated using the spatial analyst extension of the GIS software ArcView version 9.3 (ESRI, Redlands, CA). The same software was used to extract NDVI, TIR and elevation for the 220 village locations.

Colinearity between each pair of environmental variables (NDVI, TIR, elevation and distance to coastline) was assessed in Stata/SE Version 10 (Stata Corporation, College Station, TX, USA) statistical software package with 0.9 defined as the cut-off. If co-linearity was observed separate models incorporating the different variables would be constructed. Backwards step-wise regression analysis was conducted on the remaining variables to determine their inclusion into the final spatial 
prediction model. Those variables with a $p$-value $<0.1$ were retained.

\section{Bayesian geo-statistical model}

A spatial prediction model (Figure 3) was constructed based on the principle of model-based geostatistics [29] in the Bayesian statistical software WinBUGS version 14.1 (MRC Biostatistics Unit, Cambridge, UK). The model comprises two components: a deterministic component consisting of village-level fixed effects; and a stochastic component consisting of an isotropic, stationary autocorrelation function describing village-level spatial variation (i.e. a geostatistical random effect). To predict the prevalence at unsampled locations, a grid was generated of 1958 prediction locations with a spacing of 0.01 decimal degrees (approximately $1 \mathrm{~km}$ ), covering the island. Using the in-built spatial.unipred function in WinBUGS, the geostatistical random effect was interpolated to all prediction locations and predicted prevalence was calculated by adding the random effect to the sum of the products of the coefficients for the covariates and the values of the covariates at each prediction location.

\section{Model validation}

Validation of predicted prevalence was undertaken by partitioning the data into four random subsets, running the model using three of the four subsets and validating the model with the remaining subset. Four separate models were run, each with a different subset excluded for val-

Assume that the number found positive for malaria parasitaemia at location $i$ is $Y_{i}$ out of $N_{i}$ examined then $Y_{i}$ is a binomial random variable:

$$
Y_{i} \approx \operatorname{Bin}\left(N_{i}, p_{i}\right)
$$

The bivariate ordinary logistic regression model is given by:

$$
\log \left(\frac{p_{i}}{1-p_{i}}\right)=\beta_{0}+\beta_{1 \text { env }}
$$

Where $\beta_{o}$ is the intercept, env $_{i}$ is the environmental covariate, $\beta_{1}$ is the corresponding regression parameter. The spatial correlation is modelled by inclusion of a random effect $S_{i}$ :

$$
\log \left(\frac{p_{i}}{1-p_{i}}\right)=\beta_{0}+\beta_{1} e n v_{i}+S_{i}
$$

The spatial component, $S_{i}$, is defined by the isotropic, exponentially decaying correlation function:

$$
f\left(d_{i j} ; \phi\right)=\exp \left[-\left(\phi \cdot d_{i j}\right)\right]
$$

The range $\Phi$ measures the rate of decay of spatial autocorrelation, and $d_{i, j}=x_{i}-x_{j}$ measures the Euclidian distance between locations $x_{i}$ and $x_{j}$. idation. The accuracy of the prediction was determined in terms of area under curve (AUC) of the receiver-operating characteristic (ROC), with observed prevalence, dichotomised at 0 and $\geq 0$, taken as the comparator. This gave an indication of the ability of the model to discriminate between areas where transmission did and did not occur. As a general rule, an AUC between 0.5 and 0.7 indicates a poor discriminative capacity; 0.7-0.9 indicate a reasonable capacity; and $>0.9$ indicate a very good capacity.

\section{Results}

The overall prevalence of $P$. falciparum and $P$. vivax infection was $1.0 \%$ (95\% CI: $0.79-1.21 \%)$ and $2.2 \%$ (95\% CI: 1.06-3.34\%) respectively [25] with the spatial distribution broadly similar between the species (Figure 2).

Semivariograms (Figure 2) revealed spatial autocorrelation was a feature of raw prevalence of $P$. vivax and $P$. falciparum. Colinearity of 0.91 (measured using Pearson's correlation coefficient) was observed between elevation and proximity to coastline. NDVI and TIR were not statistically significant predictors and thus were not included in the final models. Exclusion of the four aforementioned villages did not affect the significance of environmental variables and thus a separate model was not deemed necessary.

Tables 1 and 2 present the results of the Bayesian geostatistical models for $P$. vivax and $P$. falciparum. For $P$. vivax, the model with distance to coastline as the fixed effect gave the best fit with better predictive ability and lower mean error and mean square errors values than the model with elevation as the fixed effect. For $P$. falciparum, the model with elevation as fixed effect gave the best fit. On the basis of the models and validation it was possible to predict the distributions of $P$. vivax and $P$. falciparum risk across the island (Figure 4 and Figure 5 respectively). The strong relationship between proximity to coastline and $P$. vivax gave a smooth risk map with endemicity classes following the contour of the coastline. The risk maps for $P$. vivax and $P$. falciparum include the upper and lower Bayesian credible limits and show the extent of uncertainty in predicted malaria risk in 2008.

\section{Discussion}

The Bayesian prediction models clearly show that transmission is not homogeneous, with malaria risk displaying a predominately coastal distribution, concentrated within well-delimited foci or 'hot-spots'. While the presence of malaria foci on the island is supported by anecdotal evidence the presence of an inland 'hot-spot' in the Green Hill area is contentious. An entomological survey carried out on the island concurrently with the parasitological survey did not reveal any vector breeding sites in the Green Hill area and concluded that transmission is 
Table 1: Results of Bayesian geostatistical models to predict prevalence of $P$. vivax and $P$. falciparum for Tanna Island, 2008.

$\begin{array}{ll}\text { Coefficient, posterior meant } & \begin{array}{l}\text { Odds ratio, posterior mean (95\% } \\ \text { Bayes credible intervals\#) }\end{array}\end{array}$

\section{Model of $P$. vivax with distance to coastline fixed effect}

\begin{tabular}{|c|c|c|c|}
\hline$a$ (intercept) & $-4.680(-5.317-4.137)$ & & \\
\hline Distance from coastline (OR per $1 \mathrm{~km}$ ) & $-0.690(-1.151-0.242)$ & $0.730(0.591-0.895)$ & \\
\hline$\Phi$ (rate of decay of spatial correlation)* & 251.5(51.26-569.4) & & \\
\hline$\sigma^{2}$ (variance of geostatistical random effect) ${ }^{* *}$ & $0.214(0.056-0.624)$ & & \\
\hline$D I C$ & & & 306.9 \\
\hline \multicolumn{4}{|l|}{ Model of $P$. vivax with elevation fixed effect } \\
\hline$a$ (intercept) & $-4.611(-5.327--3.931)$ & & \\
\hline Elevation (OR per 100 m) & $-0.547(-0.992-0.115)$ & $0.654(0.468,0.917)$ & \\
\hline$\Phi$ (rate of decay of spatial correlation)* & $167.8(33.71,461.2)$ & & \\
\hline$\sigma^{2}$ (variance of geostatistical random effect) $)^{* *}$ & $2.471(1.271,4.304)$ & & \\
\hline
\end{tabular}

DIC

Model of P. falciparum with distance to coastline fixed effect

\begin{tabular}{lll}
\hline$a$ & $-5.238(-6.027--4.625)$ & \\
\hline Distance from coastline (OR per $1 \mathrm{~km})$ & $-0.101(-0.534-0.334)$ & $0.955(0.783,1.165)$ \\
\hline$\Phi$ & $289.5(51.61-575.4)$ & \\
\hline$\sigma^{2}$ & $0.584(0.057-4.713)$ & \\
\hline DIC & & 219.5
\end{tabular}

Model of P. falciparum with elevation fixed effect

$a$

$-5.129(-5.976--4.416)$

Elevation (OR per $100 \mathrm{~m}$ )

$-0.207(-0.673-0.146)$

$0.864(0.603,1.149)$

$\Phi$

$238.2(53.28-478.9)$

$\sigma^{2}$

$1.753(0.4521-4.079)$

DIC

* The unit is change in spatial autocorrelation per decimal degree. A lower $\Phi$ indicates that spatial correlation occurs over longer distances (i.e. spatial clusters are larger).

${ }^{* *}$ A higher variance indicates a greater tendency toward spatial clustering.

\# Bayes credible intervals can be interpreted as having a similar meaning to confidence intervals used in frequentist statistics. 
Table 2: Summary of validation statistics for the geostatistical models described in Table 1.

\begin{tabular}{|c|c|c|c|}
\hline Model & AUC & Mean Error\# (\% prevalence) & Mean Absolute Error* (\% prevalence) \\
\hline Model of $P$. vivax with distance to coastline fixed effect & 0.867 & 5.07 & 1.30 \\
\hline Model of $P$. vivax with elevation fixed effect & 0.857 & 5.46 & 1.33 \\
\hline $\begin{array}{l}\text { Model of P. falciparum with distance to coastline fixed } \\
\text { effect }\end{array}$ & 0.821 & 0.39 & 0.55 \\
\hline Model of $P$. falciparum with elevation fixed effect & 0.856 & 0.34 & 0.50 \\
\hline
\end{tabular}

restricted to within $2 \mathrm{~km}$ of the coast. It appears that the Green Hill area, which is approximately $5 \mathrm{~km}$ from the coast, does not support the local vector and that malaria infections were contracted whilst inhabitants visited coastal areas. The Green Hill area focus requires further investigation, particularly with respect to patterns of mobility for work and recreation. In addition, a better understanding of the transmission patterns could be obtained from surveying Anopheles farauti breeding sites on the island during the dry season.

The risk maps have been used to define zones within which interventions are scaled and planned according to malaria transmission intensity. This will involve indoor residual spraying (IRS) on the coastal fringe but not the hinterland/middle bush area. Additionally the maps provide the base for the design of the surveillance strategy and will be fully implemented by targeted surveys which will in turn inform modifications in local implementation policies such as inclusion of a particular inland hotspot for IRS.

Whilst the maps of the upper and lower Bayesian credible intervals are important for scientific honesty (i.e. highlighting areas where the predictions are imprecise) they are also a useful tool for priority setting and thus aiding in the sound and rational deployment of interventions. The maps showing the lower Bayesian credible intervals are particularly useful in highlighting those locations in which there is high certainty of above-average malaria prevalence. Active surveillance within highprevalence foci is a cornerstone for success in interrupting malaria transmission [30]. The upper Bayesian credible intervals are useful in providing an indication of the maximum extent of malaria transmission or risk.

At the community level the risk maps have been highly successful in planning meetings. The importance of grassroots-level involvement and local ownership of the elimination goal is well recognized [2]. The island has had a long history of malaria control and such maps are an effective way for local citizens to visualize and understand the strategy for the elimination programme.

Initial statistics analysis of environmental covariates revealed no association between malaria risk and NDVI or TIR. At the small geographical scale of our study (550 $\mathrm{km}^{2}$ ), NDVI and TIR are relatively homogeneous and thus not indicative of the presence or absence of malaria. NDVI has been positively associated with malaria prevalence throughout Africa $[8,18,22,31,32]$ and the Middle East [14], but these studies covered wide geographical areas exhibiting substantial variations in climate. The relatively small size of the study area meant that the commonly used source of vegetation cover, Globcover [33], was not available at a small enough resolution to usefully define vector habitats of $A n$. farauti.

The present study found $P$. vivax risk to be associated with proximity to the coastline, which has been identified by others as a desirable $A n$. farauti habitat [34,35]. It is believed the relatively small number of $P$. falciparum cases detected on the island were not sufficient to yield conclusive results concerning associations with environmental variables. Thus despite both $P$. vivax and $P$. falciparum being supported by the same vector species slightly different relationships with environmental variables were observed.

As the need to define the limits of malaria distribution and to predict the distribution within these limits increases, the utility of ecological and climatic variables in aiding this endeavour have been questioned. While an issue that cannot be resolved within this brief discussion suffice to say that as vectors' biological niches continue to be determined by ecological and climatic factors (along with human related socio-economic factors) understanding and identifying these variables will continue to be 


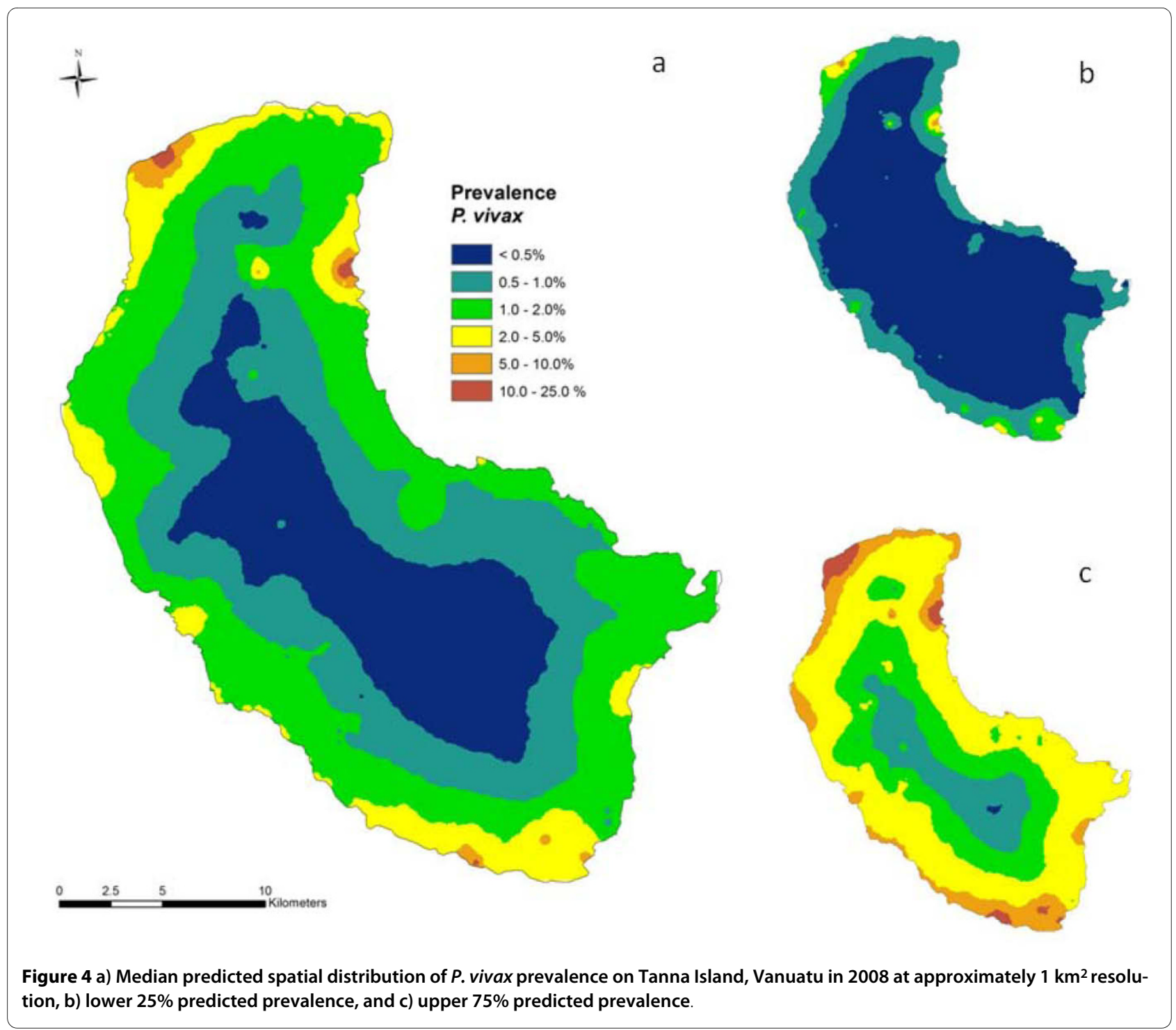

important. However, at differing spatial scales and for different vector species the significance of ecological and climatic variables will vary. For example, increases in altitude are commonly associated with cooler temperatures and thus a less suitable environment for malaria transmission for much of Africa [36-38], while in the forested hills of South-East Asia increased altitude has been found to be associated with increased forest cover and thus a more suitable transmission environment for the vector species in this ecological niche $[39,40]$. Looking at smaller spatial scales, variables such distance to local water bodies and a households' location with respect to the village periphery become important determinants of malaria risk $[10,12]$.

Accurate data on distribution of population across the island was not available at the time of this study but has been identified as a priority for the malaria elimination programme. However, the population is described to be is spread over the entire island with an inland plateau region more densely populated [25]. Once more accurate population data is available a better understanding of disease burden is possible.

While these maps will serve as baseline maps for the elimination programme such intensive surveying (i.e. $76 \%$ of all children aged 2-12 years [25]) will not be necessary to update the maps. As surveillance operations become more streamlined the routine data collected from peripheral aid posts can be used. Additionally, the data collected throughout the year can be adjusted for seasonality [24] to provide more accurate estimates of average annual prevalence.

The current study does come with some limitations, particularly the geo-referencing method, which was applied retrospectively using independently sourced coordinates (rather than during the survey using a global positioning system). Additionally, the cross-sectional sur- 


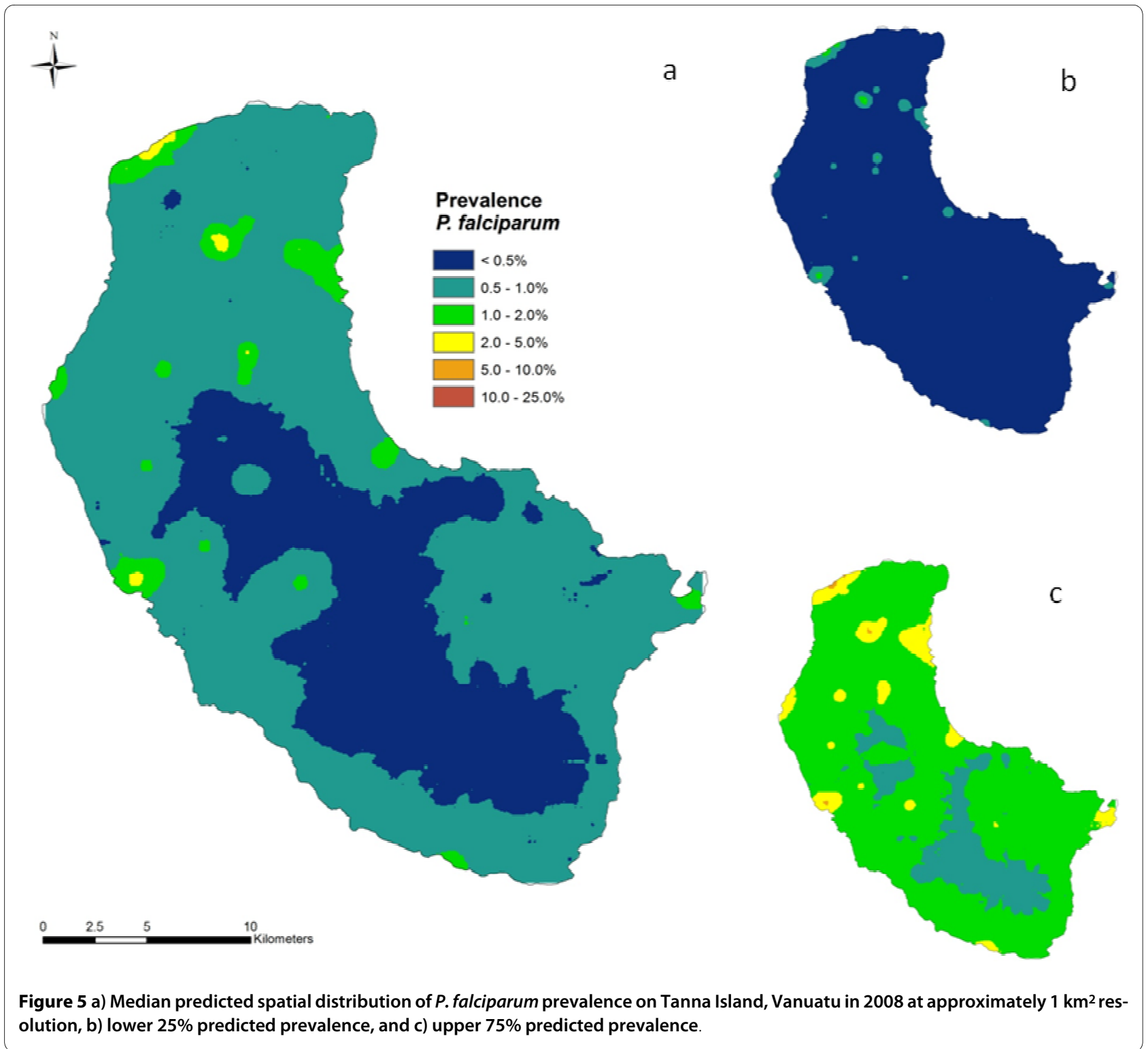

vey design meant that prevalence data were obtained at a single time point and thus the data only represent a snapshot of malaria risk, which is known to have important temporal dynamics. Despite these limitations, as data are updated with the input from surveillance operations, the spatial predictions presented here represent the baseline of a dynamic, and ideally shrinking, malaria risk map for Tanna Island.

\section{Competing interests}

The authors declare that they have no competing interests.

\section{Authors' contributions}

PaCMISC, AMI and the VBDCP conceived the study and participated in its design and data collection. AMI assembled the bulk of the incidence data. AC and HR devised and implemented the analytical methods. AT provided processed satellite images for analysis. HR wrote the first draft of the manuscript.
All authors participated in the interpretation of results and in the writing and editing of the manuscript.

\section{Acknowledgements}

Our special thanks go to the people of Tafea Province who participated in the study and without whom this research would not have been possible. We thank our colleagues at the National Vector Borne Disease Control Program, Vanuatu, (particularly Jenifer lavro, Malao John Kalomuana and Ken Mera) and at the Australian Army Malaria Institute in Brisbane (especially Christine Atkins, Alison Auliff, Lisa Bain, Qin Cheng, Robert Cooper, Andrew Ebringer, Mike Edstein, Nathan Elmes, Karen-Ann Gray, John Humphries, Marie-Louise Johnson, Darren Krause, Ken Lilley, Brady McPherson, Rob Perrin, Wesley Sharrock, John Staley and Norman Waters), for conducting the field survey in Tafea and for providing the malaria point prevalence data that was used to develop the risk maps presented in this paper. We would additionally like to thank Marcel Tanner and Dennis Shanks from the AusAID Malaria Reference Group for their guidance and mentorship. AJT is supported by a grant from the Bill and Melinda Gates Foundation (\#49446). This work forms part of the output of the Pacific Malaria Initiative principally funded by AusAID. 


\section{Author Details}

1Pacific Malaria Initiative Support Centre (PacMISC), Australian Centre for International and Tropical Health (ACITH), School of Population Health, University of Queensland, Queensland, Australia, 2National Vector Borne Disease Control Program (VBDCP), Ministry of Health, Port Vila, Vanuatu, ${ }^{3}$ Australian Army Malaria Research Institute, Department of Defence, Government of Australia, Queensland, Australia, ${ }^{4}$ Emerging Pathogens Institute and Department of Geography, University of Florida, Gainesville, USA and ${ }^{5}$ Australian Centre for Tropical and International Health, Queensland Institute of Medical Research, Brisbane, Queensland, Australia

Received: 2 December 2009 Accepted: 2 June 2010

Published: 2 June 2010

\section{References}

1. Feachem RA, Phillips A, Targett G: Shrinking the malaria map: A prospectus on malaria elimination San Francisco: The Malaria Elimination Group: 2009.

2. Kaneko G, Taleo M, Kalkoa S, Yamar T, Kobayakawa A, Björkman A: Malaria eradication on islands. Lancet 2000, 356:1560-1564.

3. Sabot O, Tulloch J, Basu S, Dyckman W, Moonasar D, Moonenf B: Getting to Zero. In Shrinking the malaria map: A prospectus on elimination Edited by: Feachem RGA, Phillips AA, Targett GA. San Francisco: The Global Health Group; 2009

4. Greenwood BM: Control to elimination: implications for malaria research. Trends Parasitol 2008, 24:449-454.

5. Carter R, Mendis KN, Roberts D: Spatial targeting of interventions against malaria. Bull World Health Organ 2000, 78:1401-1411.

6. Ernst K, Adoka S, Kowuor D, Wilson M, John C: Malaria hotspot areas in a highland Kenya site are consistent in epidemic and non-epidemic years and are associated with ecological factors. Malar J 2006, 5:78.

7. Brooker S, Clements A, Hotez P, Hay S, Tatem A, Bundy D, Snow R: The codistribution of hookworm and Plasmodium falciparum among African schoolchildren. Malar J 2006, 5:99.

8. Omumbo JA, Hay SI, Snow RW, Tatem AJ, Rogers DJ: Modelling malaria risk in East Africa at high-spatial resolution. Trop Med Int Health 2005, 10:557-566.

9. Kreuels B, Kobbe R, Adjei S, Kreuzberg C, von Reden C, Bater K, Klug S, Busch W, Adjei O, May J: Spatial variation of malaria incidence in young children from a geographically homogeneous area with high endemicity. J Infect Dis 2008, 197:85-93.

10. Myers W, Myers A, Cox-Singh J, Lau H, Mokuai B, Malley R: Microgeographic risk factors for malarial infection. Malar J 2009, 8:27.

11. Kazembe L, Kleinschmidt I, Holtz T, Sharp B: Spatial analysis and mapping of malaria risk in Malawi using point-referenced prevalence of infection data. Int J Health Geogr 2006, 5:41.

12. Cohen JM, Ernst K, Lindblade KA, Vulule JM, Chandy CJ, Wilson ML: Topography-derived wetness indices are associated with householdlevel malaria risk in two communities in the western Kenyan highlands. Malar J 2008, 7:

13. Basáñez M, Marshall C, Carabin H, Gyorkos T, Joseph L: Bayesian statistics for parasitologists. Trends Parasitol 2004, 20:85-91.

14. Brooker S, Leslie T, Kolaczinski K, Mohsen E, Mehboob N, Saleheen S, Khudonazarov J, Freeman T, Clements A, Rowland M, Kolaczinski J: Spatial epidemiology of Plasmodium vivax, Afghanistan. Emerg Infect Dis 2006, 12:1600-1602

15. Kleinschmidt I, Sharp BL, Clarke GPY, Curtis B, Fraser C: Use of generalized linear mixed models in the spatial analysis of small-area malaria incidence rates in KwaZulu Natal, South Africa. Am J Epidemiol 2001, 153:1213-1221.

16. Gemperli A, Vounatsou P, Sogoba N, Smith T: Malaria mapping using transmission models: application to survey data from Mali. Am J Epidemiol 2006, 163:289-297.

17. Noor AM, Clements A, Gething PW, Moloney G, Borle M, Shewchuk T, Hay SI, Snow RW: Spatial predition of Plasmodium falciparum prevalence in Somalia. Malar J 2008, 7 :

18. Kleinschmidt I, Bagayoko M, Clarke GP, Craig M, le Sueur D: A spatial statistical approach to malaria mapping. Int J Epidemiol 2000, 29:355-361.

19. Kleinschmidt I, Sharp B, Mueller I, Vounatsou P: Rise in malaria incidence rates in South Africa: A small-area spatial analysis of variation in time trends. Am J Epidemiol 2002, 155:257-264.
20. Gemperli A, Sogoba N, Fondjo E, Mabaso M, Bagayoko M, Briet OJT, Liebe $J$, Anderegg D, Smith T, Vounatsou P: Mapping malaria transmission in West and Central Africa. Trop Med Int Health 2006, 11:1032-1046.

21. Kleinschmidt I, Omumbo J, Briët O, van de Giesen N, Mensah NK, Windmeijer P, Moussa M, Teuscher T: An empirical malaria distribution map for West Africa. Trop Med Int Health 2001, 6:779-786.

22. Gosoniu L, Vounatsou P, Sogoba N, Maire N, Smith T: Mapping malaria risk in West Africa using a Bayesian non-parametric non-stationary model. Comput Stat Data Anal 2009, 53:3358-3371.

23. Guerra C, Gikandi P, Tatem A, Noor A, Smith D, Hay S, Snow R: The limits and intensity of Plasmodium falciparum transmission: implications for malaria control and elimination worldwide. PLoS Med 2008, 5:e38.

24. Hay SI, Guerra CA, Gething PW, Patil AP, Tatem AJ, Noor AM, Kabaria CW, Manh BH, Elyazar IRF, Brooker S, Smith DL, Moyeed RA, Snow RW: A World Malaria Map: Plasmodium falciparum endemicity in 2007. PloS Med 2009, 6:e1000048.

25. Melanesian survey team: Malaria on isolated melanesian islands prior to the initiation of malaria elimination activities. Mal J in press.

26. United States Geological Survey (USGS) Earth Resources Observation and Science Center [http://glovis.usgs.gov]

27. Tucker C: Red and photographic infrared linear combinations for monitoring vegetation. Rem Sens Env 1979, 8:127-150

28. Consultative Group on International Agricultural Research Consortium for Spatial Information [http://srtm.csi.cgiar.org/]

29. Diggle PJ, Tawn JA, Moyeed R: Model-based geostatistics. J App/ Stat 1998, 47:299-350.

30. WHO: Guidelines on the elimination of residual foci of malaria transmission World Health Organization; 2007.

31. Gosoniu L, Vounatsou P, Sogoba N, Smith T: Bayesian modelling of geostatistical malaria risk data. Geospat Health 2006, 1:127-139.

32. Hay S, Snow R, Rogers D: Predicting malaria seasons in Kenya using multitemporal meteorological satellite sensor data. Trans Roy Soc Trop Med Hyg 1998, 92:12-20.

33. Global Rural-Urban Mapping Project (GRUMP) [Alpha version] Socioeconomic Data and Applications Center, Columbia University.

34. Belkin JN: The mosquitoes of the South Pacific (Diptera, Culicidae) Berkeley and Los Angeles: University of California Press; 1962.

35. Sweeney AW, Beebe NW, Cooper RD, Bauer JT, Peterson AT: Envionmental factors associated with the distribution and range limits of malaria vector Anopheles farauti in Australia. J Med Entomol 2006, 43:1068-1075

36. Drakeley C, Carneiro I, Reyburn H, Malima R, Lusingu J, Cox J, Theander T, Nkya WMM, Lemnge MM, Riley EM: Altitude-dependent and independent variations in Plasmodium falciparum prevalence in northeastern Tanzania. J Infect Dis 2005, 191:1589-1598.

37. Lindsay SW, Martens WJM: Malaria in the African highlands: past, present and future. Bull World Health Organ 1998, 76:33-45.

38. Hay SI, Omumbo J, Craig M, Snow RW: Earth observation, geographic information systems and Plasmodium falciparum malaria in subSaharan Africa. Adv Parasitol 2000, 47:173-215.

39. Das NG, Talukdar PK: Epidemiological and entomological aspects of malaria in forest-fringed villages of Sonitpur district, Assam. J Vector Borne Dis 2004, 41:5-9.

40. Erhart A, Thang ND, Hung NQ, Toi LV, Hung LX, Tuy TQ, Cong LD, Speybroeck N, Coosemans M, D'Alessandro U: Forest malaria in Vietnam: a challenge for control. Am J Trop Med Hyg 2004, 70:110-118.

doi: $10.1186 / 1475-2875-9-150$

Cite this article as: Reid et al., Baseline spatial distribution of malaria prior to an elimination programme in Vanuatu Malaria Journal 2010, 9:150 